\title{
Mastering TAPP inguinal hernia repair-tips and tricks
}

\author{
Benedetto lelpo \\ General Surgery Department, HPB unit, University Hospital Parc Salut Mar, Barcelona 08001, Spain. \\ Correspondence to: Dr. Benedetto lelpo, General Surgery Department, HPB unit, University Hospital Parc Salut Mar, Passeig
} Marítim 25-29, Barcelona 08001, Spain. E-mail: ielpo.b@gmail.com

How to cite this article: lelpo B. Mastering TAPP inguinal hernia repair-tips and tricks. Mini-invasive Surg 2021;5:10. http://dx.doi.org/10.20517/2574-1225.2021.01

Received: 6 Jan 2021 First Decision: 27 Jan 2021 Revised: 1 Feb 2021 Accepted: 3 Feb 2021 Published: 9 Mar 2021

Academic Editor: William W. Hope Copy Editor: Xi-Jun Chen Production Editor: Yue-Yue Zhang

\begin{abstract}
Laparoscopic minimally invasive surgery is increasing, and in the last decade some modifications of the technique have been introduced, especially concerning mesh type, fixation, and peritoneal closure, which are herein individually discussed. Currently, a standard unique technique is still missing, and modifications of the technique might be useful in challenging cases, such as the use of fibrine glue to both fix the mesh and close the peritoneum. The aim of this technical note essay is to discuss and update some tips and tricks as well as recent modifications of the trans-abdominal preperitoneal (TAPP) repair of groin hernia.
\end{abstract}

Keywords: Inguinal hernia, trans-abdominal preperitoneal, laparoscopic surgery

\section{INTRODUCTION}

Over the last decade, laparoscopic inguinal hernia repair has gained worldwide popularity due to several advantages, in particular the faster recovery and reduced postoperative pain compared to the open approach with superior cost-effectivity ${ }^{[1-3]}$. Since the description of the laparoscopic trans abdominal preperitoneal (TAPP) repair, the technique has undergone several modifications, such as the mesh type and fixation and the method to approximate the peritoneum, with the aim of making the procedure easier and improving results ${ }^{[3-5]}$. These modifications have also been included to face some challenging cases where the standard procedure cannot be applied. Currently, it is important that training surgeons master these modifications and the technique, including some tips and tricks, which is the aim of this technical note essay.

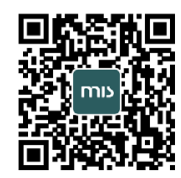




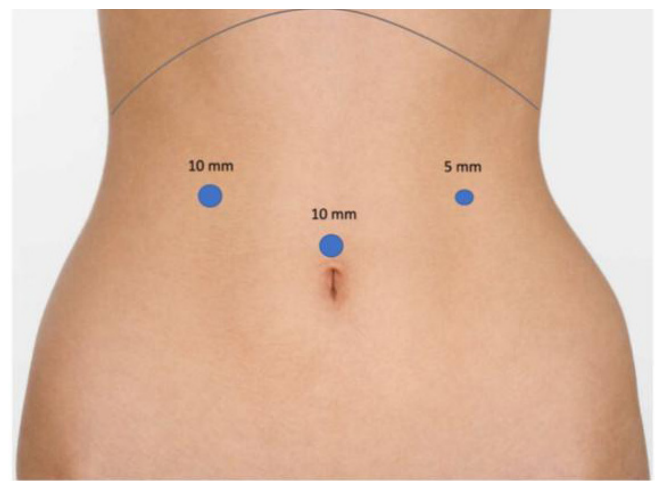

Figure 1. Trocars placement.

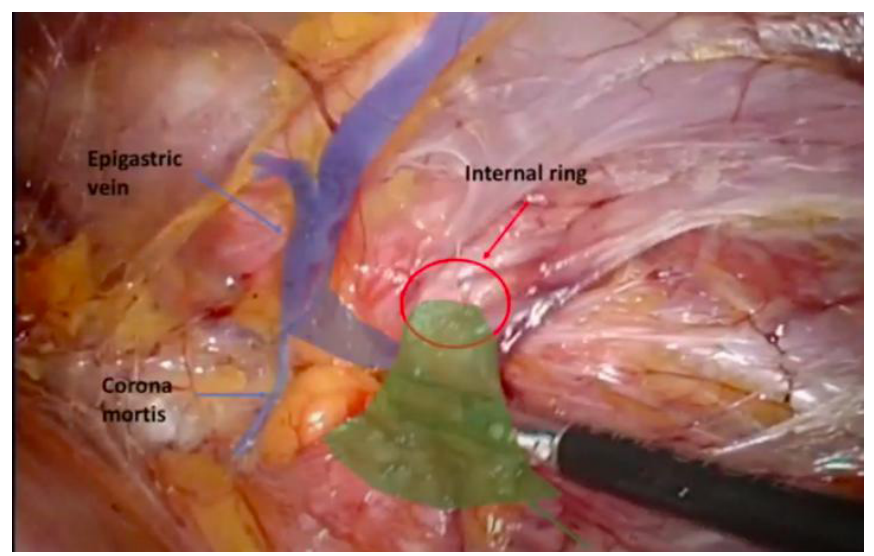

Figure 2. Surgical field.

\section{Surgical technique at our center}

A single dose of first-generation cephalosporin is given at the induction of anesthesia. The operation is performed under general anesthesia with the pneumoperitoneum established through a Veress needle in the left subcostal space, as has been previously described ${ }^{[3]}$. Three trocars are placed, as shown in Figure 1 . The peritoneum is opened approximately at the level of the lateral trocar and extended medially in the direction of the superior margin of the internal inguinal ring, up to the residue of the umbilical artery. When the Cooper ligament is exposed, the hernia sac is isolated and reduced, freeing the spermatic cord [Figure 2]. The entire video can be viewed at: https://youtu.be/6EIILTdWhoI.

\section{Postoperative pain: the main issue of laparoscopic inguinal hernia repair}

Persistent postoperative pain after placement of staples to secure the mesh, along with the discovery of the "triangle of doom" and "triangle of pain", have led to the recommendation of using only a few staples or replacing them with glue ${ }^{[2]}$. This eliminates the risk of lateral cutaneous femoral nerve entrapment, which is the main cause of chronic pain. The same suggestions are extended to the closure of the peritoneum, replacing staples as much as possible with suture or glue ${ }^{[2]}$. These modifications might increase the immediate costs. However, apart from the clinical advantages, these may entail some cost savings in the long term that only a real cost-effectiveness analysis can detect.

The type of mesh used, its fixation, and the peritoneal closure for the TAPP technique are still some of the most important topics under discussion, as several modifications of the procedure have been proposed since its first description ${ }^{[2-5]}$. 

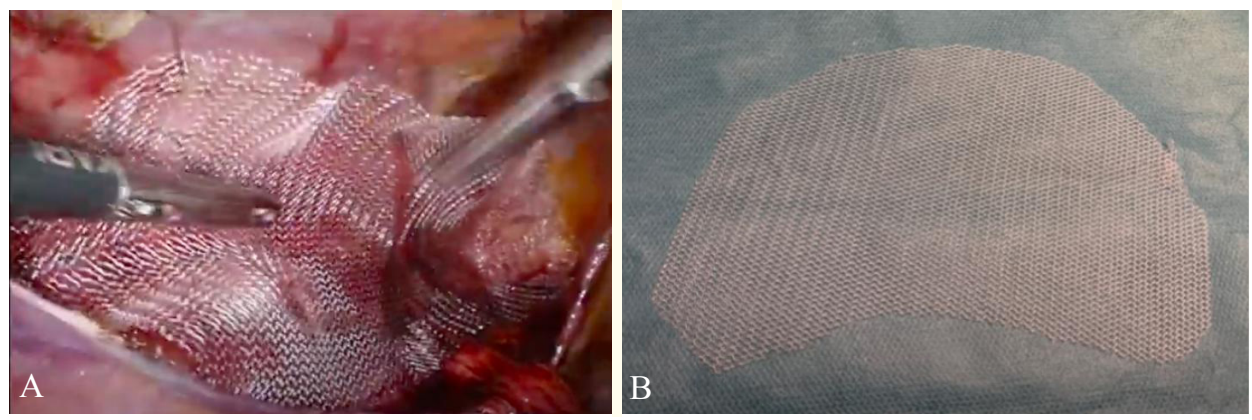

Figure 3. Self-cut polypropylene mesh placement (A, B).

\section{Mesh type}

Several meshes are available for minimally invasive inguinal hernia repair ${ }^{[4,5]}$. The surgeon must consider several factors when choosing a mesh for hernia repair including clinical outcomes, cost, and ease of use.

There are specific laparoscopic meshes with a rigid border that facilitates their placement in the preperitoneal space. However, the issue with these meshes is their size, as sometimes they do not fit within our dissection area. It happens that the surgeon is forced to enlarge the preperitoneal space to place the mesh. Another issue is its higher cost, which, for certain health systems, cannot be afforded.

Self-fixating meshes are also available and very useful, as no further fixations tools are required. However, its placement is not easy, which represents the main reason for its low application among surgeons.

Sometimes, it happens that we work in a hospital without these types of meshes. Therefore, it is important to deal with the classical mesh, used for the open approach, and cut them to be adapted for the laparoscopic technique.

In this latter case, our suggestion is to use a polypropylene mesh and to cut it into a shape of almost 10-15 $\mathrm{cm}$ to be introduced into the abdominal cavity in the preperitoneal space, as shown in Figure 3.

\section{Fixation of the mesh}

Several randomized studies have shown that using staples for mesh fixation might cause high early postoperative pain and chronic pain ${ }^{[6,7]}$. Therefore, it is suggested to minimize their use as much as possible by applying only one staple to the Cooper ligament or by using self-fixating meshes ${ }^{[2,6,7]}$.

Currently, there is not enough evidence to avoid fixing the mesh in the preperitoneal space, as increasing numbers of recurrences have been described ${ }^{[3]}$.

Some authors have reported their experience avoiding the use of staples and securing the mesh with glue only, such as fibrine glue or cyanoacrylate ${ }^{[7,8]}$.

Mesh fixation with fibrine glue was proven to be safe and effective in the prospective randomized trial of Lovisetto et al. ${ }^{[s]}$, published more than a decade ago, and it was associated with a lower incidence of postoperative neuralgia compared with staples.

In light of these results, after some time, we modified our TAPP technique where staples were used for both mesh and peritoneal closure to avoid staples altogether and replace them with fibrine glue to fix the mesh. 


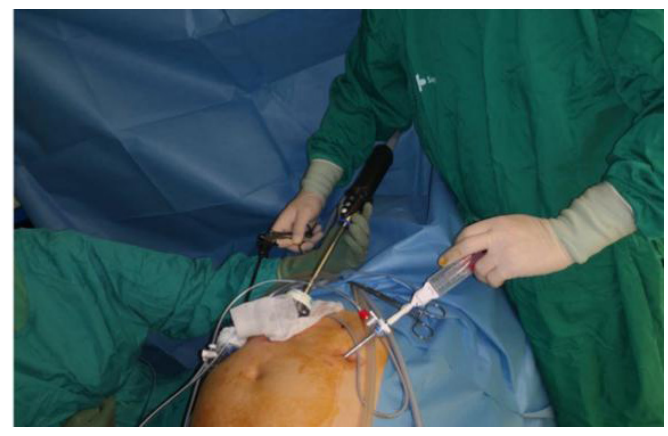

Figure 4. Fibrine glue specific tool placement.

The mesh is secured with approximately $2 \mathrm{~mL}$ of fibrine glues (Tisseel, Baxter Healthcare), applied as shown in Figure 4, at almost $1 \mathrm{~cm}$ distance from the mesh, using a specific laparoscopic tool.

This tip is very useful especially in those cases where a non-specific laparoscopic mesh is used, as their structure does not fit in the preperitoneal space as with the specific mesh type.

\section{Peritoneal closure}

The original TAPP technique includes staples to both fix the mesh and close the peritoneum. This might be the fastest and easiest method, but at the price of a higher risk of nerve injury and bleeding, since staples may damage nerves and vessels ${ }^{[9]}$. To decrease chronic pain, some absorbable staples have been introduced. However, the potential decrease of bleeding cannot be avoided.

Currently, the most frequently used modification is that of the running suture to close the peritoneum. However, suturing the peritoneum is not as easy as it seems; it remains a challenging maneuver, requiring specific surgical skills to avoid tears or ruptures that may expose the mesh to the intestine, with secondary obstruction or fistulation. With the attempt to further decrease the difficulty of this procedure, barbed sutures have been introduced. However, even with this suture, it still requires some skills, and the peritoneum closure time step, even with barbed suture, may require longer time compared with the overall surgical step. With running suture, peritoneal ruptures still occur, especially in cases when the hernia sac reduction maneuver has been particularly challenging (such as with large sliding hernias, where the flap peritoneum is very thin and too weak to be closed with a suture or in cases with a large amount of fat in the peritoneum, adding difficulty to its closure for increased tension). In addition, it is important to state that running suture of the peritoneum does not avoid the risk of nerve entrapment or bleeding, as the suture of the superior flap of the peritoneum frequently includes part of the abdominal wall. Even if not well described in the literature, epigastric vessels have been frequently injured during the closing of the peritoneum. When this occurs, it is challenging to face it. For this reason, there is a need for some modifications of the technique that may not be the standard but are useful in those difficult cases.

For these cases, our specific tip is to use, when it is required, fibrine glue.

The most frequently studied glue product is N-2-butyl cyanoacrylate. It shows a great capacity for both mesh and peritoneal closure that is achieved after only a few seconds ${ }^{[10]}$. However, being a non-biological glue, one of the main criticisms is that, when this product is in contact with the intestine, strong adhesions may develop. Nevertheless, the study of Wilson et al. ${ }^{[10]}$, which recently investigated their experience with cyanoacrylate mesh and peritoneal closure, reported excellent results with no long-term complications. However, considerable precautions are required when using this product in order to avoid dropping any material into the intestine. 
An alternative to cyanoacrylate is the biological human fibrine, which has the double function of both glue and hemostatic. Different from cyanoacrylate, there is more evidence in the literature that fibrine glue may prevent peritoneal adhesions, and it may represent the optimal and safest method to close the peritoneum $^{[11]}$. We believe that the key advantage of the modified technique, where we completely replace the running suture with fibrine glue to approximate the peritoneum, is particularly useful for those cases where the peritoneum is at a higher risk of tear or rupture during closure.

According to our experience, in our previous published study, we were able to show a decreased operative time of the procedure while also maintaining acceptable postoperative outcomes and quality of life ${ }^{[3]}$. To date, this is the unique study showing long-term results using fibrine glue to both fix the mesh and close the peritoneum.

Furthermore, according to our experience, we found the peritoneal closure with fibrine glue a simple to learn and master maneuver that does not require specific skills.

\section{CONCLUSION}

\section{Tips and tricks}

Avoid use of stapler to fix the mesh and close the peritoneum.

The knowledge of some alternatives of the technique are paramount in challenging inguinal hernia repairs, for example: Peritoneal closure can be performed using glue when its closure is challenging. When a specific mesh is not available, it is paramount to know how to prepare it.

\section{DECLARATIONS}

\section{Authors' contributions}

The author contributed solely to the article.

\section{Availability of data and materials}

Not applicable.

\section{Financial support and sponsorship}

None.

\section{Conflicts of interest}

The author declared that there are no conflicts of interest.

\section{Ethical approval and consent to participate}

Not applicable.

\section{Consent for publication}

Not applicable.

\section{Copyright}

(c) The Author(s) 2021.

\section{REFERENCES}

1. Ielpo B, Nuñez-Alfonsel J, Duran H, et al. Cost-effectiveness of randomized study of laparoscopic versus open bilateral inguinal hernia repair. Ann Surg 2018;268:725-30. 
2. HerniaSurge Group. International guidelines for groin hernia management. Hernia 2018;22:1-165.

3. Ielpo B, Ferri Valentina, Silva J, et al. Laparoscopic transabdominal preperitoneal (TAPP) inguinal hernia repair using fibrin glue for fixation of the mesh and peritoneum closure. Surg Laparosc Endosc Percutan Tech 2020;30:e24-7.

4. Chen LS, Chen WC, Kang YN, Wu CC, Tsai LW, Liu MZ. Effects of transabdominal preperitoneal and totally extraperitoneal inguinal hernia repair: an update systematic review and meta-analysis of randomized controlled trials. Surg Endosc 2019;33:418-28.

5. Shi Z, Fan X, Zhai S, Zhong X, Huang D. Fibrin glue versus staple for mesh fixation in laparoscopic transabdominal preperitoneal repair of inguinal hernia: a meta-analysis and systematic review. Surg Endosc 2017;31:527-37.

6. Andresen K, Fenger AQ, Burcharth J, Pommergaard HC, Rosenberg J. Mesh fixation methods and chronic pain after transabdominal preperitoneal (TAPP) inguinal hernia surgery: a comparison between fibrin sealant and tacks. Surg Endosc 2017;31:4077-84.

7. Harsløf S, Krum-Møller P, Sommer T, Zinther N, Wara P, Friis-Andersen H. Effect of fixation devices on postoperative pain after laparoscopic ventral hernia repair: a randomized clinical trial of permanent tacks, absorbable tacks, and synthetic glue. Langenbecks Arch Surg 2018;403:529-37.

8. Lovisetto F, Zonta S, Rota E, et al. Use of human fibrin glue (Tissucol) versus staples for mesh fixation in laparoscopic transabdominal preperitoneal hernioplasty: a prospective, randomized study. Ann Surg 2007;245:222-31

9. Antoniou SA, Köhler G, Antoniou GA, Muysoms FE, Pointner R, Granderath FA. Meta-analysis of randomized trials comparing nonpenetrating vs mechanical mesh fixation in laparoscopic inguinal hernia repair. Am J Surg 2016;211:239-249.e2.

10. Wilson P, Hickey L. Laparoscopic transabdominal preperitoneal (TAPP) groin hernia repair using n-butyl-2-cyanoacrylate (Liquiband ${ }^{\mathbb{}}$ Fix $8^{\mathrm{TM}}$ ) for mesh fixation and peritoneal closure: learning experience during introduction into clinical practice. Hernia 2019;23:601-13.

11. Tavares K, Mayo J, Bogenberger K, Davis SS Jr, Yheulon C. Fibrin versus cyanoacrylate glue for fixation in laparoscopic inguinal hernia repair: a network meta-analysis and indirect comparison. Hernia 2020;24:927-35. 